\title{
Over 10 Gbps VLC for long-distance applications using a GaN-based series-biased micro-LED array
}

\author{
Enyuan Xie, Rui Bian, Xiangyu He, Mohamed Sufyan Islim, Graduate Student Member, IEEE, Cheng Chen, \\ Jonathan J. D. McKendry, Erdan Gu, Member, IEEE, Harald Haas, Fellow, IEEE, \\ and Martin D. Dawson, Fellow, IEEE
}

\begin{abstract}
By employing a GaN-based series-biased microlight emitting diode ( $\mu$ LED) array and orthogonal frequency division multiplexing modulation format, a high-speed free-space visible light communication system for long-distance applications has been demonstrated. The blue series-biased $\mu$ LED array, which consists of $3 \times 3,20 \mu$ m-diameter $\mu$ LED elements, presents promising performance with an optical power and -6dB electrical modulation bandwidth of over $10 \mathrm{~mW}$ and $980 \mathrm{MHz}$, respectively. Record data transmission rates have been successfully achieved at different free-space distances. Within $5 \mathrm{~m}$ transmission distances, over $10 \mathrm{Gbps}$ data rates at the forward error correction (FEC) floor of $3.8 \times 10^{-3}$ are accomplished. Extending the transmission distances to $20 \mathrm{~m}$, the data rates are maintained at the Gbps level at the FEC floor.
\end{abstract}

Index Terms-Long-distance visible light communication (VLC), series-biased micro-LED ( $\mu$ LED) array, orthogonal frequency division multiplexing (OFDM).

\section{INTRODUCTION}

$\mathbf{V}$ ISIBLE light communication (VLC) has emerged as a possible supplement to radio-frequency (RF) communication in recent years, driven by the increasing demands of wireless communication services and the limited availability of RF spectrum [1]. In VLC, data is transmitted by electrically modulating the optical intensity of a light source emitting in the visible range at rates unperceived by the human eye [2]. This technology offers several advantages such as license-free spectrum range, high security, and immunity to electromagnetic interference [3]. Furthermore, VLC technology can be potentially incorporated into existing solid state lighting (SSL) systems. Therefore, with the purpose of pursuing higher data transmission rate and/or longer transmission distance, much effort has been made to optimize VLC systems in terms of both transmitters [4], [5], [6] and modulation formats [7], [8].

Manuscript received April 19, 2005; revised August 26, 2015. This research was supported by Engineering and Physical Sciences Research Council (EPSRC) grant EP/M01326X/1 (QuantIC). Data is available online at https://doi.org/10.15129/ae577969-aa18-47f2-8dff-df6a20eba41e. (Corresponding author: Mohamed Sufyan Islim and Erdan Gu)

Enyuan Xie, Xiangyu He, Jonathan J. D. McKendry, Erdan Gu and Martin D. Dawson are with Institute of Photonics, Department of Physics, University of Strathclyde, Glasgow, G1 1RD, UK (e-mail: enyuan.xie@strath.ac.uk; xiangyu.he@strath.ac.uk; jonathan.mckendry@strath.ac.uk; erdan.gu@strath.ac.uk; m.dawson@strath.ac.uk).

Rui Bian, Mohamed Sufyan Islim, Cheng Chen and Harald Haas are with LiFi R\&D Centre, the University of Edinburgh, Institute for Digital Communications, Kings Buildings, Mayfield Road, Edinburgh, EH9 3JL, UK (e-mail: R.Bian@ed.ac.uk; M.islim@ed.ac.uk; Cheng.Chen@ed.ac.uk; H.Haas@ed.ac.uk).

Enyuan Xie, Rui Bian and Xiangyu He contributed equally to this work.
Regarding the transmitters, as the common inorganic light sources in SSL systems, GaN-based light emitting diodes (LEDs) are considered as the strongest candidates to enable illumination and communication applications simultaneously. However, due to the large resistance-capacitance time constant of the p-n junction, commercial GaN-based LEDs normally show a low $-6 \mathrm{~dB}$ electrical modulation bandwidth in the range of 10-20 MHz [9]. In order to overcome this limitation, microLED ( $\mu$ LED) emitters, which have lateral dimensions less than $100 \mu \mathrm{m}$, have been introduced to enable high-speed VLC systems [10]. Thanks to their small junction volume and high operating current density, $\mu$ LEDs present a $-6 \mathrm{~dB}$ electrical bandwidth up to a few hundred $\mathrm{MHz}$ [10]. On the modulation format part, although single carrier modulation formats, such as onoff keying (OOK) and pulse amplitude modulation (PAM), can be implemented straightforwardly for VLC, their performance degrades as the data transmission speed increases. This is primarily due to the low-pass characteristics of the front-ends elements, which leads to severe inter-symbol interference when excessive high symbol rate is used. A multicarrier modulation scheme, known as orthogonal frequency division multiplexing (OFDM), is able to slice the transmission channel into a large number of narrowband subchannels. This makes OFDM be able to operate in the severely non-flat channels with a single-tap equalization. Furthermore, the adaptive bit and energy loading in OFDM also allows the channel utilization to approach the information capacity limit. By using a single $\mu$ LED and OFDM modulation format, up to $7.91 \mathrm{Gbps}$ data transmission rate has been achieved at the forward error correction (FEC) floor of $3.8 \times 10^{-3}$ in our recent work [8]. However, due to the relatively low optical power of $\mu$ LEDs, the data transmission distance in this work was limited to $0.3 \mathrm{~m}$. To the best of our knowledge, using a single $\mu$ LED for long-distance VLC applications (>3 m), the reported highest data transmission rate is around $1 \mathrm{Gbps}$ at $10 \mathrm{~m}$ distance assuming OOK modulation format [11]. To realize the full potential of VLC technology and implement its applications in the fields such as Lighting-as-a-Service and the Internet of Things, a substantial increase in the VLC longdistance data transmission rate is urgently needed.

In this work, using a GaN-based series-biased $\mu$ LED array with the optimized design based on VLC link setup and OFDM modulation format, we experimentally demonstrate a highspeed VLC system for free-space long-distance applications. As a transmitter, the blue-emitting $(450 \mathrm{~nm})$ series-biased $\mu$ LED array consists of $3 \times 3,20 \mu$ m-diameter $\mu$ LED elements. 


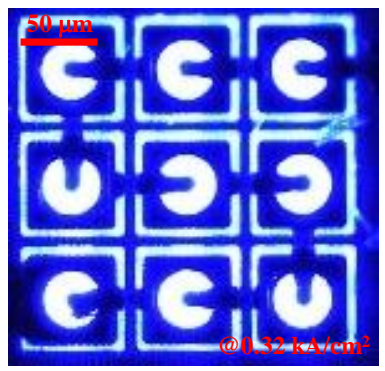

(a)

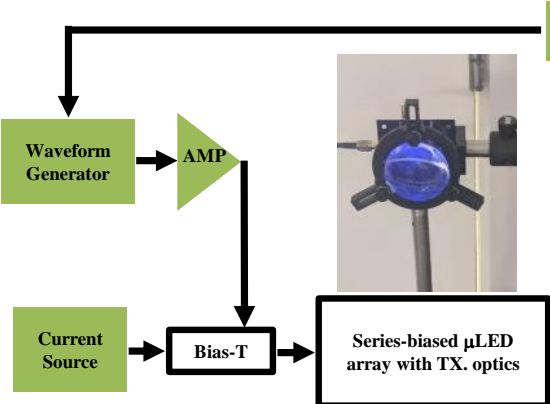

array with TX. optics

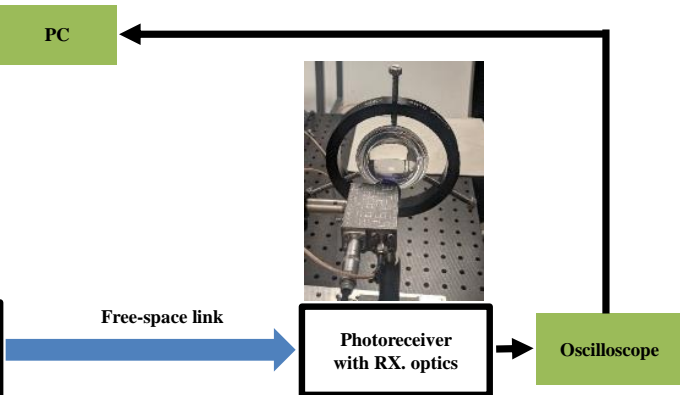

(b)

Fig. 1. (a) Plan-view optical micrograph image of the fabricated series-biased $\mu$ LED array operating at $0.32 \mathrm{kA} / \mathrm{cm}^{2}$; (b) schematic diagram of the setup for different-distance VLC measurements, the optical images of the transmitter and receiver modules are inserted as well.

This array presents over $10 \mathrm{~mW}$ optical power and $980 \mathrm{MHz}$ $-6 \mathrm{~dB}$ electrical modulation bandwidth, respectively. For VLC applications, at the FEC floor of $3.8 \times 10^{-3}$, the record data transmission rates of $11.74,11.72,10.11,6.58,2.84$ and $1.61 \mathrm{Gbps}$ are achieved at the free-space distances of 0.3 , $2,5,10,15$, and $20 \mathrm{~m}$, respectively, assuming the OFDM modulation format. The results presented in this work not only demonstrate state-of-the-art VLC data transmission rates at different distances using $\mu$ LED-based transmitters, but also show the high potential of series-biased $\mu \mathrm{LED}$ arrays for longdistance VLC applications.

\section{EXPERIMENTS}

\section{A. Design and Fabrication of the Series-biased $\mu L E D$ Array}

The series-biased $\mu$ LED arrays were fabricated from commercial blue GaN-based LED wafers grown on c-plane sapphire with periodically patterned surfaces. The detailed epitaxial structure of the wafers can be found in [6]. The seriesbiased $\mu$ LED array developed in this work comprises a $3 \times 3$ layout of disk-shape $\mu$ LED elements, each with a $20 \mu \mathrm{m}$ diameter, in a flip-chip configuration. The center-to-center pitch between two adjacent $\mu$ LED elements is $80 \mu \mathrm{m}$. The number of $\mu$ LED elements employed in array is set as 9 due to the performance limitations of the equipment used in the VLC experiments, such as the voltage of bias-T and gain of amplifier. The fabrication process of the series-biased $\mu$ LED array has been detailed described in [6]. The fabricated array was then wire-bonded to a printed circuit board for further characterizations and VLC measurements without any specific thermal managements. Fig. 1(a) shows a plan-view optical micrograph image of the fabricated series-biased $\mu$ LED array operating at $0.32 \mathrm{kA} / \mathrm{cm}^{2}$. Based on our simulation study (data not shown), compared with the devices presented in our previous work [6], the smaller element diameter and centerto-center pitch employed in this work enable not only higher modulation bandwidths but also improved light collection efficiency in the free-space links used in this work. Thanks to this improved collection efficiency, although the absolute direct-current (DC) optical power emitted from the arrays is lower, the higher channel gain of VLC link is achieved, especially for long-distance cases. These changes permit the fast data transmission rates at each experimental distance, which will be shown in Section III.

\section{B. Measurement Setup}

The room-temperature current density versus voltage $(J$ $V)$, optical power versus current density $(J-L)$ and $-6 \mathrm{~dB}$ electrical modulation bandwidth characteristics of the wirebonded series-biased $\mu \mathrm{LED}$ array were measured following the similar methods to those described in [8]. The irradiance beam profile of this $\mu$ LED array was also measured at $8.12 \mathrm{kA} / \mathrm{cm}^{2}$ operation current density and a distance of $0.3 \mathrm{~m}$ between the array and a $\mathrm{Si}$ photodetector.

Fig. 1(b) shows a schematic diagram of the setup used in this work for free-space VLC systems with different distances, assuming the DC-bias optical OFDM format. The optical images of the transmitter and receiver modules are also inserted. The distance between the series-biased $\mu$ LED array and photoreceiver was set as $0.3,2,5,10,15$, and $20 \mathrm{~m}$ by changing the position of the photoreceiver. The detailed information of the used DC-bias optical OFDM format can be found in [8]. As shown, the OFDM waveforms were generated by MATLAB ${ }^{\circledR}$ and forwarded to an arbitrary waveform generator (AWG, Kesysight M8195A). A high-power amplifier (SHF S126A) then amplified the output analogue signals from AWG. The amplified signals and a DC current density were then combined by a bias-T (Tektronix PSPL5575A) and applied to the series-biased $\mu$ LED array. After extensive experiments to optimize the influence of nonlinear distortion on the system performance, the DC-bias current density, $J_{D C}$, and modulation signal depth, $V_{P P}$, were set as $8.12 \mathrm{kA} / \mathrm{cm}^{2}$ and $8.17 \mathrm{~V}$, respectively. The light emitted from the $\mu \mathrm{LED}$ array was first collimated by an aspheric condenser lens (TX. optics; Thorlabs ACL 4532A). Another aspheric condenser lens (RX. optics; Thorlabs ACL 4532A for 0.3 and $2 \mathrm{~m}$ distances, Thorlabs ACL 7560 for 5, 10, 15, and $20 \mathrm{~m}$ distances) was employed to focus the light onto a fast-speed photoreceiver (Femto HSPR-X-I-1G4-SI). The output signals of the photodetector were captured by a digital oscilloscope (Kesysight DSA90804B) and sent back to the PC for decoding. By replacing the photoreceiver with the Si photodetector, the received optical power after lens focusing at each measurement distance was also measured.

\section{RESULTS AND DISCUSSION}

Fig. 2(a) illustrates $J-V$ and $L-J$ characteristics of the blue series-biased $\mu$ LED array used in this work. As shown, this 
array presents a promising electrical performance with a low turn-on voltage of $27.5 \mathrm{~V}$ at $0.32 \mathrm{kA} / \mathrm{cm}^{2}(1 \mathrm{~mA})$ and series resistance of $165 \Omega$ corresponding to $3.1 \mathrm{~V}$ and $18 \Omega$ for each $\mu$ LED element. In terms of directed optical power, before thermal rollover, this array produces over $10 \mathrm{~mW}$ at an operating current density of $11.15 \mathrm{kA} / \mathrm{cm}^{2}$ following a near Lambertian irradiation intensity distribution shown in Fig. 2(c). Due to such high operating current density, a high modulation bandwidth are achieved for this $\mu \mathrm{LED}$ array. As shown in Fig. 2(b), the $-6 \mathrm{~dB}$ electrical bandwidth of this array is in excess of $980 \mathrm{MHz}$ at $11.15 \mathrm{kA} / \mathrm{cm}^{2}$. Compared with the reported single $\mu$ LED element with a similar size [10], this array demonstrates higher optical power and modulation bandwidth at the same operating current density. This is mainly due to the series-connection configuration employed in this array, which not only results in a multi- $\mu$ LED-element operation to produce the high optical power but also reduces the array capacitance to increase the modulation bandwidth [12]. These novel characteristics significantly benefit VLC applications in terms of both data rate and transmission distance.

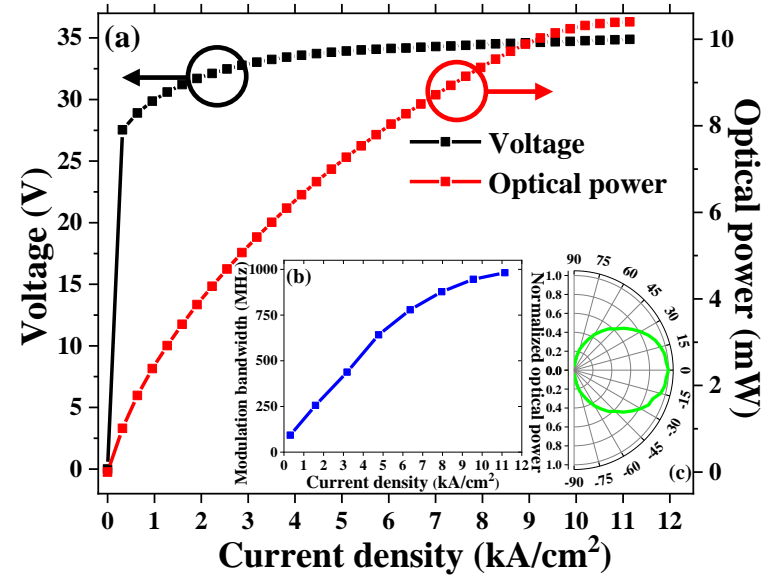

Fig. 2. (a) $J-V, L-J$ and (b) $-6 \mathrm{~dB}$ electrical bandwidth characteristics of the used blue series-biased $\mu$ LED array; (c) Normalized beam profile of the used $\mu \mathrm{LED}$ array measured at $8.12 \mathrm{kA} / \mathrm{cm}^{2}$ and a distance of $0.3 \mathrm{~m}$.

Using the experimentally optimized $J_{D C}\left(8.12 \mathrm{kA} / \mathrm{cm}^{2}\right)$ and $V_{P P}(8.17 \mathrm{~V})$, the series-biased $\mu$ LED array was operated to firstly investigate the VLC system performance at a $0.3 \mathrm{~m}$ free-space distance. Fig. 3(a) illustrates the achieved signalto-noise ratio (SNR) versus bandwidth for the system. As shown, up to about $2.3 \mathrm{GHz}$ bandwidth is accomplished with the SNR higher than $5 \mathrm{~dB}$. Such a wide bandwidth range allows a substantial number of parallel channels for bit loading in the OFDM format to approach the limitation of data capacity. To maximize the achievable data transmission rate, adaptive bit and power loading algorithm is used. The algorithm loads as many numbers of bits as possible to aggregate data transmission rate. Meanwhile, the power allocation scheme tries to adjust the SNR on subcarriers with same bit loading to a level that is just sufficient to be reliably decoded with the FEC threshold. The bit loading and power allocation per subcarrier for the OFDM signal are illustrated in Fig. 3(b). The recovered $M$-quadrature amplitude modulation (QAM) constellations up to $M=128$ are shown as the inserts of Fig. 3(a). They demonstrate the SNR is high enough to distinguish the transmitted symbols and, thus, permit a highspeed VLC system to be demonstrated. The measured data transmission rates against bit error rate (BER) at the $0.3 \mathrm{~m}$ free-space distance are shown in Fig. 4. Up to $11.74 \mathrm{Gbps}$ data transmission rate is successfully achieved at the FEC floor of $3.8 \times 10^{-3}$. To the best of our knowledge, this is the highest data transmission rate of the $\mu$ LED-based VLC system at such a free-space distance, which is about $50 \%$ higher than the value reported in [8]. This increase is mainly attributed to two factors. Firstly, the high optical power and high modulation bandwidth of the series-biased $\mu$ LED array result in an impressive SNR performance as discussed earlier. Secondly, the employed small pitch between the $\mu$ LED elements in the array and appropriate diameter of aspheric condenser lenses in the setup improve the light collection efficiency for the VLC system and, thus, guarantees a promising channel gain of VLC link. Furthermore, the bandwidth of the photoreceiver used is $1.4 \mathrm{GHz}$, which is considered as the main factor limiting the current VLC system performance. It is expected that higher data transmission rates would be achieved when a photoreceiver with higher bandwidth is employed.
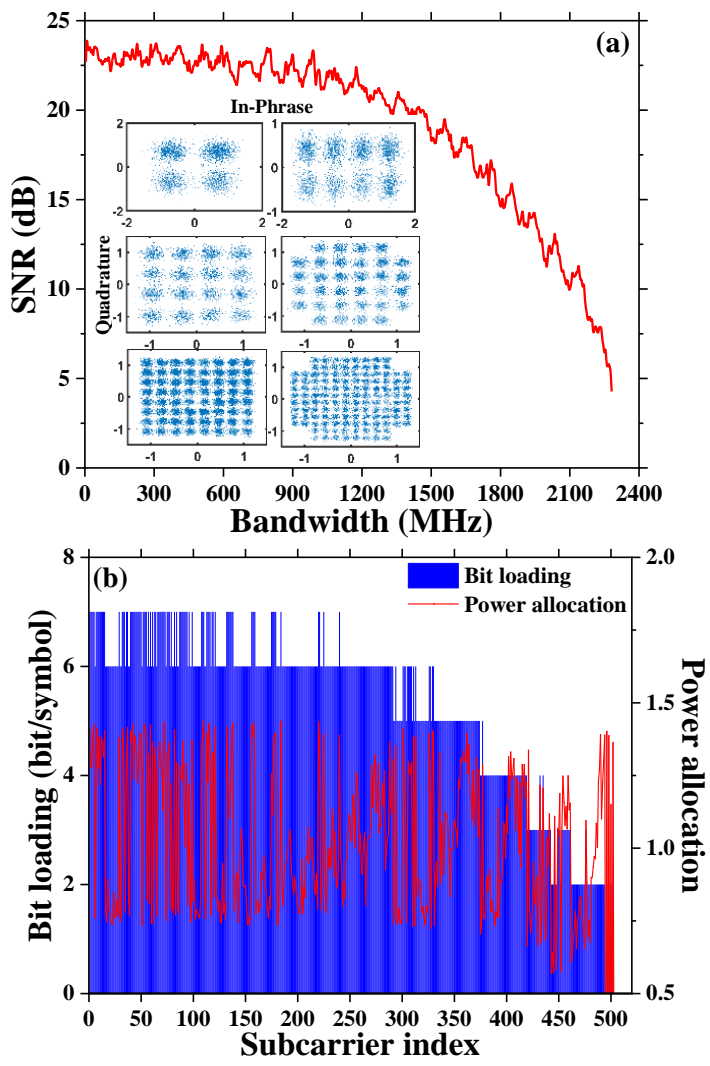

Fig. 3. (a) SNR versus bandwidth and (b) bit loading and power allocation per subcarrier for the OFDM signal for the free-space VLC system with a $0.3 \mathrm{~m}$ distance. The received $M$-QAM constellation symbols after equalization for $M=4,8,16,32,64,128$ are inserted in part (a).

In order to systemically investigate the dependence of the VLC system performance on the transmission distance, we further extended the distance between the transmitter and receiver to $2,5,10,15$, and $20 \mathrm{~m}$ respectively, for data transmission rate measurements. The data transmission rates 


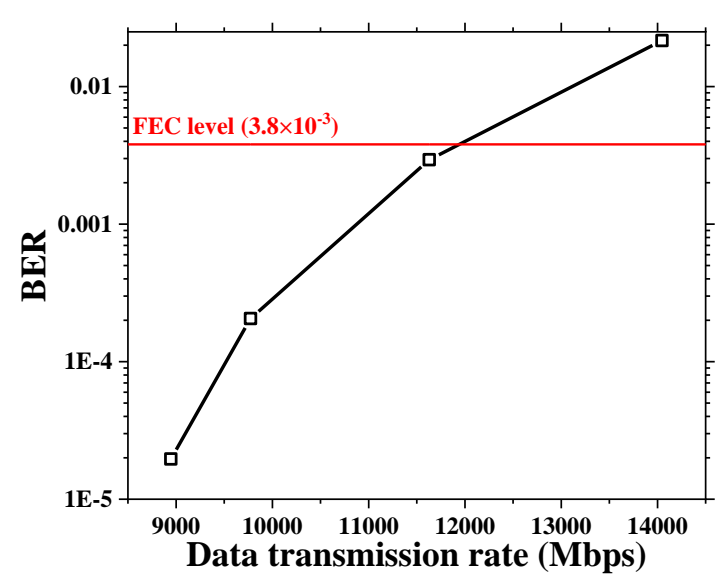

Fig. 4. BER versus data transmission rate achieved for the free-space VLC system with a $0.3 \mathrm{~m}$ distance.

achieved at the FEC floor of $3.8 \times 10^{-3}$ at these distances are summarized in Fig. 5. As shown, the data transmission rates up to $11.72,10.11,6.58,2.84$, and $1.61 \mathrm{Gbps}$ are accomplished at the distances of $2,5,10,15$, and $20 \mathrm{~m}$, respectively. The lower SNR at longer distances is considered as the main factor limiting the VLC speed. As demonstrated by the red part in Fig. 5, with the distance increasing, a sharp drop on the average SNR can be observed. This phenomenon is mainly attribute to the reduced channel gain of VLC link caused by the lower DC optical power received at longer distances, which are shown as the insert of Fig. 5. The lower SNR leads to a limitation on the channel utilization in the OFDM format and the maximum modulation order of QAM also decreases from 128 at $0.3 \mathrm{~m}$ to 16 at $20 \mathrm{~m}$. It is considered that this issue can be solved by further optimizations on the VLC optical set-up and/or adding more $\mu$ LED elements in the series-biased array. Through optimization of the VLC optical set-up, a higher channel gain of VLC link and, thus, higher SNR are expected. Meanwhile, under the same channel gain of VLC link, more $\mu$ LED elements in the series-biased array would further increase the optical-power dynamic range for bit loading and modulation bandwidth of the system. Here, it is important to indicate that, to the best of our knowledge, the achieved data transmission rates at different distances in this work represent state-of-the-art values of $\mu$ LED-based VLC systems. These results demonstrate that the series-biased $\mu \mathrm{LED}$ arrays are promising light sources for the high-speed VLC systems targeting long data transmission distances.

\section{CONCLUSION}

In conclusion, a high-speed free-space VLC system for long-distance applications has been experimentally demonstrated in this work. Using a blue series-biased $\mu$ LED array and OFDM modulation formats, up to $11.74,11.72,10.11$, $6.58,2.84$, and $1.61 \mathrm{Gbps}$ data transmission rates are achieved at the FEC floor with the distances of $0.3,2,5,10,15$, and $20 \mathrm{~m}$, respectively. The accomplishment of these state-of-theart data transmission rates is mainly attributed to the optimized design of the employed series-biased $\mu$ LED array. This array, which consists of $3 \times 3,20 \mu$ m-diameter $\mu$ LED elements with a

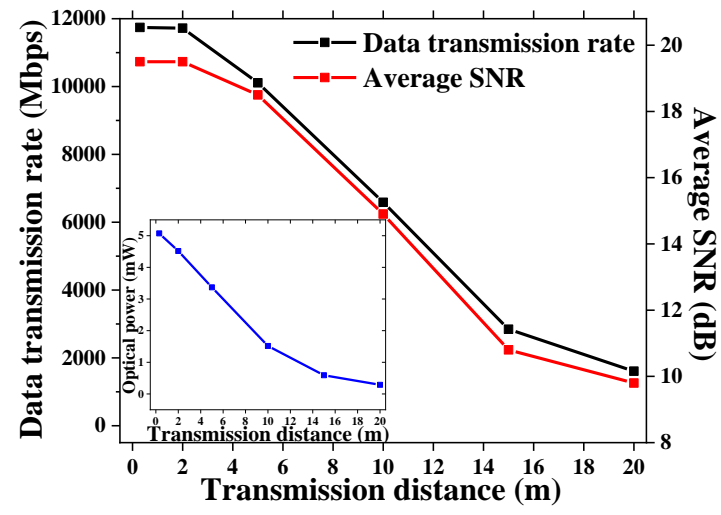

Fig. 5. Achieved data transmission rates at the FEC floor of $3.8 \times 10^{-3}$ and the average SNR at different transmission distances for the free-space VLC system. The insert shows the received DC optical power after lens focusing at different transmission distances.

$80 \mu \mathrm{m}$ pitch, not only produces an optical power over $10 \mathrm{~mW}$ accompanying with a $980 \mathrm{MHz}-6 \mathrm{~dB}$ electrical modulation bandwidth but also improves the channel gain of VLC link.

\section{REFERENCES}

[1] V. Cisco, "Cisco Visual Networking Index: Forecast and Trends, 20172022," White Paper, 2018.

[2] D. Karunatilaka, F. Zafar, V. Kalavally, and R. Parthiban, "LED based indoor visible light communications: State of the art," IEEE Communications Surveys \& Tutorials, vol. 17, no. 3, pp. 1649-1678, 2015.

[3] H. Burchardt, N. Serafimovski, D. Tsonev, S. Videv, and H. Haas, "VLC: Beyond point-to-point communication," IEEE Communications Magazine, vol. 52, no. 7, pp. 98-105, 2014.

[4] C.-L. Liao, Y.-F. Chang, C.-L. Ho, and M.-C. Wu, "High-speed GaNbased blue light-emitting diodes with gallium-doped $\mathrm{ZnO}$ current spreading layer," IEEE Electron Device Letters, vol. 34, no. 5, pp. 611-613, 2013.

[5] D. V. Dinh, Z. Quan, B. Roycroft, P. J. Parbrook, and B. Corbett, "GHz bandwidth semipolar (112- 2) InGaN/GaN light-emitting diodes," Optics letters, vol. 41, no. 24, pp. 5752-5755, 2016.

[6] E. Xie, X. He, M. S. Islim, A. A. Purwita, J. J. McKendry, E. Gu, H. Haas, and M. D. Dawson, "High-speed visible light communication based on a III-nitride series-biased micro-LED array," Journal of Lightwave Technology, vol. 37, no. 4, pp. 1180-1186, 2019.

[7] Y. Zhou, J. Zhao, M. Zhang, J. Shi, and N. Chi, "2.32 Gbit/s phosphorescent white LED visible light communication aided by twostaged linear software equalizer," in 2016 10th International Symposium on Communication Systems, Networks and Digital Signal Processing (CSNDSP). IEEE, 2016, pp. 1-4.

[8] M. S. Islim, R. X. Ferreira, X. He, E. Xie, S. Videv, S. Viola, S. Watson, N. Bamiedakis, R. V. Penty, I. H. White et al., "Towards $10 \mathrm{~Gb} / \mathrm{s}$ orthogonal frequency division multiplexing-based visible light communication using a GaN violet micro-LED," Photonics Research, vol. 5, no. 2, pp. A35-A43, 2017.

[9] J. Grubor, S. C. J. Lee, K.-D. Langer, T. Koonen, and J. W. Walewski, "Wireless high-speed data transmission with phosphorescent whitelight LEDs," in 33rd European Conference and Exhibition of Optical Communication-Post-Deadline Papers (published 2008). VDE, 2007, pp. 1-2.

[10] R. X. Ferreira, E. Xie, J. J. McKendry, S. Rajbhandari, H. Chun, G. Faulkner, S. Watson, A. E. Kelly, E. Gu, R. V. Penty et al. "High bandwidth GaN-based micro-LEDs for multi-Gb/s visible light communications," IEEE Photonics Technology Letters, vol. 28, no. 19, pp. 2023-2026, 2016.

[11] X. Liu, P. Tian, Z. Wei, S. Yi, Y. Huang, X. Zhou, Z.-J. Qiu, L. Hu, Z. Fang, C. Cong et al., "Gbps long-distance real-time visible light communications using a high-bandwidth GaN-based micro-LED," IEEE Photonics Journal, vol. 9, no. 6, pp. 1-9, 2017.

[12] Y. Huang, Z. Guo, H. Huang, and H. Sun, "Influence of current density and capacitance on the bandwidth of VLC LED," IEEE Photonics Technology Letters, vol. 30, no. 9, pp. 773-776, 2018. 